\title{
Algebraic Quantization of the Closed Bosonic String*
}

\author{
Catherine Meusburger \\ Fakultät für Physik, Universität Freiburg, \\ 79104 Freiburg, Germany ${ }^{\dagger}$ \\ and \\ Karl-Henning ReHren \\ Institut für Theoretische Physik, Universität Göttingen, \\ 37073 Göttingen, Germany
}

Dedicated to Rudolf Haag on the occasion of his 80th birthday

\begin{abstract}
The gauge invariant observables of the closed bosonic string are quantized in four space-time dimensions by constructing their quantum algebra in a manifestly covariant approach, respecting all symmetries of the classical observables. The quantum algebra is the kernel of a derivation on the universal envelopping algebra of an infinitedimensional Lie algebra. The search for Hilbert space representations of this algebra is separated from its construction, and postponed.
\end{abstract}

PACS 2001: 11.25.-w, 11.30.-j, 03.65.Fd

MSC 2000: 81R10, 81T30

\section{Introduction}

Fock space quantization of String Theory is notoriously beset with tachyons and anomalies. This fact is responsible for the need of supersymmetry and extra dimensions whose introduction and interpretation have triggered several "string revolutions" with a vast range of speculative implications [2]. Yet, these features tend to obliterate the underlying simple idea of String Theory [3. In this article, we prefer to cling to that simple idea, viewed as a

\footnotetext{
${ }^{*}$ The article is based on the diploma thesis of the first author (C.M.) [1] under the supervision of K. Pohlmeyer, Universität Freiburg, completing a project by the second author (K.-H.R.) lying dormant since around 1987.

${ }^{\dagger}$ Address after January 2002: Dept. of Mathematics, Heriot-Watt University, Riccarton, Edinburgh EH14 4AS, UK, electronic address: C.Meusburger@ma.hw.ac.uk

${ }^{\ddagger}$ Electronic address: rehren@theorie.physik. uni-goe.de
} 
model for a consistent quantum theory of extended objects with a presumed relevance to gauge theories [4, 5], without ambitions towards a Theory of Everything.

The present article demonstrates the viability (with a proviso, see below) of an alternative approach to (bosonic closed) string quantization initiated in [6] (and pursued in 17, 8, 9, 10, 11, 12, 13) which does not suffer from the drawbacks mentioned above. This approach differs in at least three essential aspects from the conventional one. The first is the interpretation and quantum theoretical implementation of the constraints [10. Second, it strives to capture the observable features of the classical theory (and only these) in terms of classically reparametrization invariant quantities (the observables of the string), and to quantize only those. A complete system of classical observables has been identified [7, 9], see Sect. 2 below.

The third substantial distinction of the present approach concerns the concept of quantization. It is understood in a purely algebraical sense by consistently promoting Poisson brackets to commutators, that is, without constructing a Hilbert space representation at the same time. The representation problem is thus detached from the construction of the quantum algebra, and this opens the possibility of finding inequivalent physical (positive energy) representations of the same quantum algebra (superselection sectors). The constraints on the classical dynamical variables [6, 8] will be implemented in terms of the appropriate eigenspaces of Casimir operators of the quantum algebra (rather than using some elimination prescription). In this way, they determine the physical representations [10, and private communication by K. Pohlmeyer].

The Poisson algebra $\mathfrak{h}$ of the classical string observables exhibits a rich algebraic structure, involving an infinite number of polynomial relations among multiple Poisson brackets of its generators [7, 8, 11]. The presence of these polynomial relations severely complicates the algebraic construction of the quantum algebra.

An intrinsic approach to algebraic quantization has been pursued in 12, 13. It assigns a quantum counterpart to each classical generator, and a quantum polynomial relation to each classical polynomial relation. One admits observable subleading terms of order $\hbar$ or higher (quantum corrections) to the relations, restricted by the grading of the classical algebra. In order to determine the quantum corrections, one requires a maximum of structural similarity with the classical algebra; in particular, the commutator of a quantum relation with an observables must not generate any new relations without a classical counterpart (the principle of correspondence) 12 .

With these postulates and Poincaré covariance as guiding principles, one proceeds degree by degree in the inherent algebraic grading. It has been 
shown [12, 13 that in $1+3$ dimensions and up to degree 5, all quantum corrections can be consistently and almost uniquely determined. Three free parameters survive a highly overdetermined non-linear system of conditions at this degree. ${ }^{1}$

It remains unsatisfactory, of course, (and impracticable on the long run), to proceed degree by degree. The aim of the present article is to establish the existence of a consistent quantization prescription to all degrees.

We succeed to do so with the only proviso that, in the final step of our construction, we extrapolate an algebraic feature of the classical algebra apparent at lower degrees $(\leq 7)$, and assume its persistence to all degrees. Sect. 6 is devoted to the discussion of this "quadratic generation property", which would follow from a structural property of the underlying (explicitly known) infinite-dimensional Lie algebra. Numerical tests of this linear problem have found their limitation due the rapid growth of this graded Lie algebra [12, while structural arguments available so far are only partial [11, 14].

We pursue an extrinsic approach, which is slightly against the spirit outlined above. The idea of such an approach is to take advantage of an embedding of the classical observables into an auxiliary "ambient" Poisson algebra in which (i) the polynomial relations among the observables are identities in terms of independent (but non-observable) variables, and for which (ii) a standard quantization procedure is available. As an analogy from mechanics, instructive for this and the following (though infinitely simpler), the reader is invited to think of the relation

$$
\{A, B\}^{2}-16 A \cdot B=0
$$

which turns into an identity if one embeds $A=P^{2}, B=Q^{2}$ into the canonical Poisson algebra generated by $P$ and $Q$. Identities in the classical ambient algebra will acquire quantum corrections of order $\hbar$ or higher in the quantized algebra.

The quantum observables are then sought as elements of the non-commutative quantized ambient algebra, e.g., by the specification of an embedding prescription (such as a suitable factor ordering) for the expression of a generating set of observables in terms of the non-observable quantized variables. Thus, classical polynomial relations involving multiple Poisson brackets of the generators naturally give rise to the corresponding polynomial relations in the quantum algebra involving multiple commutators of the generators, exhibiting quantum corrections. These quantum corrections, of course, a priori belong to the ambient algebra.

\footnotetext{
${ }^{1}$ The complexity of the problem is illustrated by the number of 106.089 relations at degree 5 among 2.337 invariants of degree $\leq 5$ (in $1+3$ dimensions) 13 .
} 
The principle of correspondence in this case stipulates that they must be quantum observables, since otherwise the quantized theory would have new observables without a classical counterpart. The problem is thus to establish the existence of an embedding prescription such that the quantum corrections to the polynomial relations are observables. Once this has been achieved, the quantum algebra of observables is well defined and obeys the principle of correspondence. The remaining challenge of finding and classifying positive energy Hilbert space representations concerns only this algebra and not the quantized ambient algebra which need not be represented.

In the standard approach, the ambient algebra is obtained by canonical quantization of the string's Fourier modes in a given parametrization. It has been shown, however, that this choice, together with frequency normal ordering, produces non-invariant quantum corrections to reparametrization invariant relations [15] (apart from the well known violation of Poincaré covariance in that approach) and thus violates the principle of correspondence.

The ambient algebra which we choose in this article, is the envelopping algebra of an infinite-dimensional Lie algebra (with respect to the Poisson bracket). Its generators are the components of an infinite set of Lorentz tensors ("monodromy variables") which depend on the string parametrization only through the choice of a reference point on the string's world surface.

The classical observables are represented as polynomials in the independent monodromy variables. It follows that multiple Poisson brackets among observables, and polynomials therein, are also polynomials in these variables. The polynomial relations defining the classical algebra of observables are, when expressed as polynomials in the monodromy variables, identities. (In fact, this is how the polynomial relations were originally derived in $[7,8,11$.)

The quantum ambient algebra is defined by promoting the classical Poisson bracket of the Lie algebra to a commutator, and passing to the universal envelopping algebra. The quantum algebra of observables is defined as the kernel of a suitable derivation, acting on this non-commutative algebra. The quantum counterparts of the classical observable generators are identified as non-commutative polynomials annihilated by the derivation. This implies that all their multiple commutators and non-commutative polynomials therein also belong to the kernel. In particular, the quantum corrections obtained by replacing in the classical polynomial relations classical generators by quantum generators, Poisson brackets by commutators, and commutative products by non-commutative ones with any choice of factor ordering, belong to the kernel and hence indeed are also observables.

This approach results in a consistent covariant quantization of the string observables in any dimension, complying with the principle of correspondence, provided we may take for granted the quadratic generation property. 


\section{Outline of the classical structure}

With the left-moving and right-moving modes $( \pm)$ of every classical solution $x_{\mu}(\tau, \sigma)$ of the closed bosonic string in $D$ space-time dimensions, one can associate two Lax pairs [6]. These are systems of linear partial differential matrix equations whose integrability conditions are equivalent to the Nambu-Goto equation of motion. They involve $D$ arbitrary $n \times n$ matrices $T^{\mu}, \mu=0 \ldots D-1$, of arbitrary size $n$. The spectra of the corresponding monodromy matrices $\phi_{T}^{ \pm}$are reparametrization invariant non-local functionals of the world surface. The observables of the classical bosonic string are obtained by variation with respect to the parameter matrices.

We describe the salient algebraic structures of the classical observables (invariants). More detailed formula will be given in Sect. 4.

\subsection{Invariants [6, 7, 9]:}

Since the size $n$ of the parameter matrices $T^{\mu}$ is arbitrary, there are infinitely many free parameters. Varying $\operatorname{Tr}\left(\phi_{T}^{ \pm}-\mathbf{1}_{n}\right)=\sum_{N=1}^{\infty} \mathcal{Z}_{\mu_{1} \ldots \mu_{N}}^{ \pm} \operatorname{Tr} T^{\mu_{1}}$ $\cdots T^{\mu_{N}}$ with respect to the matrices $T^{\mu}$, one obtains two infinite systems of reparametrization invariant observables $\mathcal{Z}_{\mu_{1} \ldots \mu_{N}}^{ \pm}$(henceforth called invariants). They are explicitly given as iterated integrals over left- and right-moving modes of the canonical string variables, $u_{\mu}^{ \pm}(\tau, \sigma)=p^{\mu} \pm \partial_{\sigma} x^{\mu}$,

$$
\mathcal{Z}_{\mu_{1} \ldots \mu_{N}}^{ \pm}=\mathfrak{z}_{N} \circ \int_{\sigma<\sigma_{N}<\cdots<\sigma_{1}<\sigma+2 \pi} d \sigma_{1} \cdots d \sigma_{N} \quad u_{\mu_{1}}^{ \pm}\left(\tau, \sigma_{1}\right) \cdot \ldots \cdot u_{\mu_{N}}^{ \pm}\left(\tau, \sigma_{N}\right)
$$

where $\mathfrak{z}_{N}$ denotes the sum over the cyclic permutations of the Lorentz indices. They do not depend on the choice of the reference point $(\tau, \sigma)$ in the formula.

The invariants are reparametrization invariant functionals of the string's world surface. Their values, together with the infinitesimal generators of Lorentz boosts, locally determine the world surface up to translations in the direction of the string's momentum.

With respect to their canonical Poisson bracket (see below) and their multiplication as functionals they form a Poisson algebra, the classical algebra of observables. The invariants $\mathcal{Z}_{\mu_{1} \ldots \mu_{N}}^{+}$and $\mathcal{Z}_{\mu_{1} \ldots \mu_{N}}^{-}$associated with left and right moving modes, respectively, constitute two Poisson commuting subalgebras $\mathfrak{h}^{+}$and $\mathfrak{h}^{-}$which are isomorphic up to a global sign in the structure constants.

The invariants are Lorentz tensors $\mathcal{Z}_{\mu_{1} \ldots \mu_{N}}^{ \pm}$of any rank $N \geq 1$. The vector invariants $\mathcal{P}_{\mu}=\mathcal{Z}_{\mu}^{+}=\mathcal{Z}_{\mu}^{-}$(arising at first order in $T^{\mu}$ ) are the components of the total momentum of the string. These are the only invariants which are common to both systems $\mathfrak{h}^{ \pm}$of invariants. The remaining invariants of either system are algebraically independent of those of the other 
system, and each system involves infinitely many algebraically independent ones. Thus, the quantum algebra $\widehat{\mathfrak{h}}^{(-)}$will be the opposite algebra of $\widehat{\mathfrak{h}}^{(+)}$. In the sequel, we describe the left-moving one, and omit the distinguishing superscript + .

2.2. Monodromy variables (truncated tensors) [6, 7, 9]:

In order to analyse the structure of the classical algebra of observables, the invariants were expressed as polynomials in another set of Lorentz tensors, called truncated tensors in [7]. In the following, we shall prefer the term monodromy variables. They are generated by the logarithm $\log \Phi_{T}(\tau, \sigma)=\sum_{N=1}^{\infty} \mathcal{R}_{\mu_{1} \ldots \mu_{N}}^{t}(\tau, \sigma) T^{\mu_{1}} \cdots T^{\mu_{N}}$ of the monodromy matrix, which (unlike the trace) depends on the choice of a reference point $(\tau, \sigma)$ on the string's world surface. Therefore, the monodromy variables $\mathcal{R}_{\mu_{1} \ldots \mu_{N}}^{t}$ are not completely reparametrization invariant. Their dependence on the string coordinates is given by

$$
\begin{aligned}
& \partial_{\sigma} \mathcal{R}_{\mu_{1} \ldots \mu_{N}}^{t}(\tau, \sigma)=u_{\mu_{1}}(\tau, \sigma) \mathcal{R}_{\mu_{2} \ldots \mu_{N}}^{t}(\tau, \sigma)-u_{\mu_{N}}(\tau, \sigma) \mathcal{R}_{\mu_{1} \ldots \mu_{N-1}}^{t}(\tau, \sigma) \\
& \partial_{\tau} \mathcal{R}_{\mu_{1} \ldots \mu_{N}}^{t}(\tau, \sigma)=\gamma(\tau, \sigma) \partial_{\sigma} \mathcal{R}_{\mu_{1} \ldots \mu_{N}}^{t}(\tau, \sigma)
\end{aligned}
$$

where $u_{\mu}(\tau, \sigma)$ are the canonical string variables and $\gamma$ is an arbitrary function, which reflects the choice of a gauge.

The monodromy variables $\mathcal{R}_{\mu_{1} \ldots \mu_{N}}^{t}$ at any fixed point $(\tau, \sigma)$ are algebraically independent functionals up to linear relations involving sums over "shuffle permutations" of their indices according to eq. (4.2) below.

The bracket among the monodromy variables induced by the canonical bracket between left and right moving modes violates the Jacobi identity due to the singular behaviour of the canonical bracket. But it can be modified so as to restore the Jacobi identity, and without affecting the Poisson bracket among the invariants (which are polynomials in the monodromy variables) [7, p. 615]. In compact notation for the generating functionals, the (modified) Poisson bracket is given by [16]

$$
\begin{aligned}
& \left\{\log \Phi_{S}{ }^{\otimes}, \log \Phi_{T}\right\}= \\
& \quad=\left.\operatorname{Tr}\left([S \cdot T, S-T] \cdot \partial_{X}\right) \log \Phi_{X}\right|_{X=S+T}-\left[S \cdot T, \log \Phi_{S}-\log \Phi_{T}\right]
\end{aligned}
$$

where the matrices $S^{\mu}$ and $T^{\mu}$ are understood as $S^{\mu} \otimes \mathbf{1}$ and $\mathbf{1} \otimes T^{\mu}$, and the differential operator $\operatorname{Tr}\left(Y \cdot \partial_{X}\right)$ acts as the substitution of $Y^{\mu}$ for $X^{\mu}$ in a derivational manner. For a more explicit formula in terms of the components, see eq. (4.3) below. Equipped with this bracket, the linear span $\Re$ of the monodromy variables $\mathcal{R}_{\mu_{1} \ldots \mu_{N}}^{t}$ at any fixed point $(\tau, \sigma)$ is an infinitedimensional Lie algebra. Its symmetric envelopping algebra $\mathcal{S}(\mathfrak{R})$ is therefore canonically a Poisson algebra. 
The classical invariants are the reparametrization invariant polynomials in the monodromy variables $\mathcal{R}_{\mu_{1} \ldots \mu_{N}}^{t}(\tau, \sigma)$, characterized by the condition

$$
\partial_{\sigma} \mathcal{Z}=0
$$

Each of them is a linear combination of tensor components $\mathcal{Z}_{\mu_{1} \ldots \mu_{N}}$ of the form given in eq. (4.4). Their linear span $\mathfrak{h}$ is in fact closed under multiplication and under Poisson brackets (extended to $\mathcal{S}(\mathfrak{R})$ by the Leibniz rule). In other words, $\mathfrak{h}$ is the Poisson subalgebra of $\mathcal{S}(\mathfrak{R})$ given by

$$
\mathfrak{h}=\operatorname{Ker} \partial_{\sigma} \subset \mathcal{S}(\mathfrak{R})
$$

\subsection{Homogeneity and grading [7]:}

The Poisson algebra $\mathcal{S}(\mathfrak{R})$ possesses two gradings: one with respect to the order $N \geq 1$ in the spectral parameters $T^{\mu}$ (i.e., the total Lorentz tensor rank disregarding possible contractions), and one with respect to the order $K \geq 1$ as a polynomial in the monodromy variables.

Both the rank and the order are additive with respect to the product, whereas the Poisson bracket reduces the total rank by two and the total order by 1 . The two gradings can be unified to a single grading with respect to the degree $l=N-K-1 \geq-1$, which is additive under the Poisson bracket whereas the product of two elements of degrees $l$ and $l^{\prime}$ is of degree $l+l^{\prime}+1$ :

$$
\mathcal{S}(\mathfrak{R})=\bigoplus_{l \geq-1} \mathcal{S}(\mathfrak{R})^{(l)}, \quad \begin{array}{ll}
\mathcal{S}(\mathfrak{R})^{(l)} \cdot \mathcal{S}(\mathfrak{R})^{\left(l^{\prime}\right)} \subset \mathcal{S}(\mathfrak{R})^{\left(l+l^{\prime}+1\right)} \\
& \left\{\mathcal{S}(\mathfrak{R})^{(l)}, \mathcal{S}(\mathfrak{R})^{\left(l^{\prime}\right)}\right\} \subset \mathcal{S}(\mathfrak{R})^{\left(l+l^{\prime}\right)} .
\end{array}
$$

The spaces $\mathcal{S}(\mathfrak{R})^{(l)}$ are spanned by spanned by all monomials of order $K$ in components of $\mathcal{R}_{\mu_{1} \ldots \mu_{N}}^{t}$ with total tensor rank $N$ and $N-K-1=l$.

The homogeneous parts and the parts of fixed tensor rank of each invariant polynomial in the monodromy variables are separately invariant. The gradings of $\mathcal{S}(\mathfrak{R})$ therefore give rise to gradings of $\mathfrak{h}$. In particular, with $\mathfrak{h}^{(l)}=\mathfrak{h} \cap \mathcal{S}(\mathfrak{R})^{(l)}$, one has the grading with respect to the degree

$$
\mathfrak{h}=\bigoplus_{l \geq-1} \mathfrak{h}^{(l)}, \quad \begin{array}{ll}
\mathfrak{h}^{(l)} \cdot \mathfrak{h}^{\left(l^{\prime}\right)} \subset \mathfrak{h}^{\left(l+l^{\prime}+1\right)} \\
\left\{\mathfrak{h}^{(l)}, \mathfrak{h}^{\left(l^{\prime}\right)}\right\} \subset \mathfrak{h}^{\left(l+l^{\prime}\right)}
\end{array}
$$

The subalgebra $\mathcal{S}(\mathfrak{R})^{(-1)}=\mathfrak{h}^{(-1)}$ consists of the polynomials in the components of the total momentum $\mathcal{P}_{\mu}=\mathcal{R}_{\mu}^{t}=\mathcal{Z}_{\mu}$. Except for this algebra of central elements acting on each of them as multipliers, the subspaces $\mathcal{S}(\mathfrak{R})^{(l)}$ and $\mathfrak{h}^{(l)}$ are finite-dimensional. 
The components $\mathcal{P}_{\mu}$ are central in the Lie algebra $\mathfrak{R}$ and hence in the Poisson algebra $\mathfrak{h}$. As a function on the classical phase space, $\mathcal{P}_{\mu}$ takes values in the closed forward light-cone, with light-like values only on rather singular configurations. In the sequel, we shall regard the mass square $\mathcal{P}_{\mu} \mathcal{P}^{\mu}$ as a number $m^{2}$, i.e., each algebra is tacitly understood as a quotient by the relation $\mathcal{P}_{\mu} \mathcal{P}^{\mu}=m^{2}$, and $m^{2}$ is assumed $>0 .^{2}$

The invariants of degree $l=0$ act by Poisson brackets on $\mathfrak{h}$ as infinitesimal Lorentz transformations belonging to the stabilizer subgroup $\mathrm{SO}(3)$ of the vector $\mathcal{P}_{\mu}$. Thus the invariants of fixed degree can be classified according to the representations (multiplets) of $\mathrm{SO}(3)$ and parity, which considerably facilitates the investigation of the algebra [12].

\subsection{Generating invariants and polynomial relations [7]:}

An algebraic basis of $\mathfrak{h}$ containing infinitely many algebraically independent invariants has been identified [7, Sect. IV] with the aid of the monodromy variables. However, it turns out that there is no algebraic basis of invariants whose linear span is a Lie algebra. Instead, the Poisson bracket between two basis invariants generically is a polynomial in basis invariants. Hence the Poisson algebra $\mathfrak{h}$ of classical string observables is not the symmetric envelopping algebra of a Lie algebra.

The grading of $\mathfrak{h}$ with respect to the degree $l$ suggests investigating its generation via the Poisson bracket, such that every invariant can be represented as a polynomial in the generating invariants and their multiple Poisson brackets. It is known that, as a Poisson algebra, $\mathfrak{h}$ is not generated by its elements of lowest degrees $l \leq 1$ [7, p. 622]. But there is considerable evidence (for $m^{2}>0$, and at least in $1+2$ and $1+3$ dimensions [11, 12, 14]) that it can be generated by elements which are quadratic in the monodromy variables. We shall take this quadratic generation property (hypothesis) for granted in the final step of our construction (Prop. 4), and refer to Sect. 6 for a discussion of this issue.

More specifically, in $1+3$ dimensions, the generating invariants consist of the multiplet $J_{1}$ of generators of the little group $S O(3)$ (degree 0$)$, three multiplets $S_{1}, S_{2}, T_{2}$ of degree 1 and an infinite series $\mathcal{B}_{(l)}$ of Poisson commuting Lorentz scalars, one at each odd degree $l$. All these generators are quadratic polynomials in the monodromy variables.

The generators do not generate $\mathfrak{h}$ freely. There is an infinite number of polynomial relations among multiple Poisson brackets of generating in-

\footnotetext{
${ }^{2}$ The reason for excluding the massless case (for the time being) is that in order to generate the Poisson algebra (Sect. 2.4 below), it is sometimes necessary to divide by $m^{2}$. (One may as well add an element $m^{-2}$ to the algebra $\mathcal{S}(\Re)$ which satisfies the obvious relations.) All formulae derived in the center of mass system in the previous literature can then be easily cast into covariant form. For a manifestly covariant treatment, see [17.
} 
variants. These relations are identities in $\mathcal{S}(\mathfrak{R})$ (i.e., as polynomials in the monodromy variables, and also as functionals of the canonical string variables). In particular, they are homogeneous in the degree.

The polynomial relations constitute a Poisson ideal $\mathfrak{I}$ in the symmetric envelopping algebra $\mathcal{S}(\mathfrak{F})$ of the free Lie algebra $\mathfrak{F}$ of the generators. The classical algebra of string observables $\mathfrak{h}$ is the quotient of $\mathcal{S}(\mathfrak{F})$ by this ideal. The knowledge of the ideal $\mathfrak{I} \subset \mathcal{S}(\mathfrak{F})$ (or a set of generating elements) therefore determines $\mathfrak{h}$.

\subsection{Additional structure [8, 12]:}

A maximal subalgebra $\mathfrak{A} \subset \mathfrak{h}$ of invariants with vanishing Poisson brackets has been identified in [8]. It is generated by a Cartan subalgebra of the degree 0 invariants and infinitely many Lorentz (pseudo) scalars. The latter are obtained by varying the generating functionals $\operatorname{Tr}\left(\log \phi_{\lambda \Gamma}\right)^{K}$, where $T^{\mu}=\lambda \Gamma^{\mu}$ and $\Gamma^{\mu}$ a matrix representation of the Lorentzian Clifford algebra in $D$ dimensions, with respect to the single parameter $\lambda$. Pseudoscalars arise only in odd space-time dimensions.

The scalar generators $\mathcal{B}_{(l)}$ belong to this abelian algebra $\mathfrak{A}$. The following remarkable feature has been observed. Let $\mathfrak{U}$ be the subalgebra of $\mathfrak{h}$ generated by the (finite) set of the remaining generators $J_{1}, S_{1}, S_{2}, T_{2}$. If $m^{2}>0$, the generators $\mathcal{B}_{(l)}$ can be modified to generators $\mathcal{B}_{0}^{(l)}$ within $\mathfrak{A}$ in such a way that their linear span $\mathfrak{a} \subset \mathfrak{A}$, acting by Poisson brackets on $\mathfrak{U}$, takes $\mathfrak{U}$ into itself,

$$
\{\mathfrak{a}, \mathfrak{U}\} \subset \mathfrak{U} \text {. }
$$

This observation has been verified in $d=1+3$ [1] and $d=1+2$ (unpublished) by explicit calculations up to considerable degree, but a complete proof is lacking due to the highly non-trivial combinatorics involved.

Assuming that these properties are confirmed at all degrees, then the Poisson ideal $\mathfrak{I} \subset \mathcal{S}(\mathfrak{F})$, which determines the classical algebra $\mathfrak{h}$ as discussed in Sect. 2.4, acquires further structure. It is generated by 12

- the Poisson ideal in the symmetric envelopping algebra of the free Lie algebra with finitely many generators $J_{1}, S_{1}, S_{2}, T_{2}$ which determines the Poisson algebra $\mathfrak{U}$. This ideal can be given by a set of generating polynomial relations.

- the relations stating the Poisson commutativity of the linear space $\mathfrak{a}$.

- the commutative Poisson action of $\mathfrak{a}$ on the Poisson algebra $\mathfrak{U}$, defining a semidirect product $\mathfrak{a} \ltimes \mathfrak{U} \subset \mathfrak{h}$. The corresponding ideal is generated by the Poisson brackets between the basis elements $\mathcal{B}_{0}^{(l)}$ of $\mathfrak{a}$ and the generators $J_{1}$, $S_{1}, S_{2}, T_{2}$ of $\mathfrak{U}$, taking values in $\mathfrak{U}$.

The details of the general structures outlined above may depend on the space-time dimension $D$. They can be found in the given references. The 
relevant ones will be introduced more explicitly in the next sections.

\section{Algebraic quantization: the general strategy}

\subsection{The intrinsic approach to string quantization}

The intrinsic approach to the quantization of the string observables, advocated by Pohlmeyer et al. [11, 12, 13, consciously avoids all reference to non-invariant monodromy variables $\mathcal{R}^{t}$ and proceeds directly from the Poisson algebra of classical invariants $\mathfrak{h}$ or equivalently, from the Poisson ideal $\mathfrak{I} \subset \mathcal{S}(\mathfrak{F})$ defining it.

The polynomial nature of the relations generating the ideal $\mathfrak{I}$ gives rise to non-trivial self-consistency problems in the course of quantization: the non-commutative quantum algebra should be the quotient of the universal (i.e., non-commutative) envelopping algebra $\mathcal{U}(\widehat{\mathfrak{F}})$ of the free Lie algebra $\widehat{\mathfrak{F}}$ of the quantum generating invariants by the ideal of the quantized polynomial relations. This quantum ideal should exhibit a maximum of structural similarity with the classical ideal.

Thus, in order to define the quantum algebra, the infinite set of generating quantized polynomial relations has to be specified in the universal envelopping algebra $\mathcal{U}(\widehat{\mathfrak{F}})$. These may differ from the classical generating relations only by quantum corrections of lower degree, expressible in terms of quantum generators (respecting spin and parity). The quantum corrections are strongly constrained by the postulate (principle of correspondence), that commutators between quantum relations and quantum generators must not produce new quantum relations (of order $\hbar$ ) without a classical counterpart.

This, completely intrinsic, strategy has been pursued in refs. 11, 12, 13. and has been shown to be self-consistent in a highly non-trivial manner up to degree five (in $1+3$ dimensions).

\subsection{The new approach to string quantization}

We propose, in order to quantize $\mathfrak{h}$, to quantize in a first step the Lie algebra $\mathfrak{R}$ of monodromy variables by replacing the Poisson bracket (4.3) by $i \hbar$ times the commutator, and its symmetric envelopping algebra $\mathcal{S}(\mathfrak{R})$ by the universal envelopping algebra $\mathcal{U}(\widehat{\Re})$ of the quantized Lie algebra $\widehat{\Re}$. The grading of $\mathcal{S}(\Re)$ by the degree does not survive the quantization because an evaluated commutator has lower degree than the corresponding difference of products (i.e., quantum corrections have lower degree). The remnant of the grading $\mathcal{S}(\mathfrak{R})=\bigoplus_{l} \mathcal{S}(\mathfrak{R})^{(l)}$ is thus a filtration

$$
\mathcal{U}(\widehat{\mathfrak{R}})=\bigcup_{l \geq-1} \mathcal{U}(\widehat{\mathfrak{R}})^{(l)}, \quad \begin{array}{ll}
\mathcal{U}(\widehat{\mathfrak{R}})^{(l)} \cdot \mathcal{U}(\widehat{\mathfrak{R}})^{\left(l^{\prime}\right)} \subset \mathcal{U}(\widehat{\mathfrak{R}})^{\left(l+l^{\prime}+1\right)} \\
{\left[\mathcal{U}(\widehat{\mathfrak{R}})^{(l)}, \mathcal{U}(\widehat{\mathfrak{R}})^{\left(l^{\prime}\right)}\right] \subset \mathcal{U}(\widehat{\mathfrak{R}})^{\left(l+l^{\prime}\right)} .}
\end{array}
$$


$\mathcal{U}(\widehat{\mathfrak{R}})^{(l)}$ is spanned by all non-commutative monomials of order $K$ in components of $\mathcal{R}_{\mu_{1} \ldots \mu_{N}}^{t}$ with total tensor rank $N$ and $N-K-1 \leq l$. The inclusions $\mathcal{U}(\widehat{\Re})^{(l-1)} \subset \mathcal{U}(\widehat{\Re})^{(l)}$ take care of the ambiguities concerning the grading of quantum polynomials when relations $[A, B]=A B-B A$ are taken into account.

The quantum algebra $\widehat{\mathfrak{h}}$ of the invariants will be a subalgebra of $\mathcal{U}(\widehat{\mathfrak{R}})$ with a corresponding filtration through $\widehat{\mathfrak{h}}^{(l)}=\widehat{\mathfrak{h}} \cap \mathcal{U}(\widehat{\mathfrak{R}})^{(l)}$,

$$
\widehat{\mathfrak{h}}=\bigcup_{l \geq-1} \widehat{\mathfrak{h}}^{(l)}, \quad \widehat{\mathfrak{h}}^{(l-1)} \subset \widehat{\mathfrak{h}}^{(l)}, \quad \begin{aligned}
& \widehat{\mathfrak{h}}^{(l)} \cdot \widehat{\mathfrak{h}}^{\left(l^{\prime}\right)} \subset \widehat{\mathfrak{h}}^{\left(l+l^{\prime}+1\right)} \\
& {\left[\widehat{\mathfrak{h}}^{(l)}, \widehat{\mathfrak{h}}^{\left(l^{\prime}\right)}\right] \subset \widehat{\mathfrak{h}}^{\left(l+l^{\prime}\right)} .}
\end{aligned}
$$

For the purpose of constructing this subalgebra $\widehat{\mathfrak{h}}$, it is not sufficient to specify the quantum counterparts of the generating invariants as elements of $\mathcal{U}(\widehat{\mathfrak{R}})$ such that they coincide with the classical invariants in $\mathcal{S}(\mathfrak{R})$ as the factor ordering and quantum corrections of order $\hbar$ are ignored in $\mathcal{U}(\mathfrak{\Re})$. The difficulty is due to the polynomial relations among the quantum generating invariants (and their commutators), which must hold in $\widehat{\mathfrak{h}}$. Since $\widehat{\mathfrak{h}} \subset \mathcal{U}(\widehat{\Re})$, they must be identities in $\mathcal{U}(\widehat{\Re})$. But by construction, they hold in $\mathcal{U}(\widehat{\Re})$ only up to quantum corrections of order $\hbar$ or higher. The principle of correspondence would be violated unless these quantum corrections are also polynomials in the quantum generators. Otherwise there would be elements of the quantum algebra without classical counterpart. To implement this property required by the principle of correspondence, using an ansatz for the quantum corrections to the quantum generators, would require to actually compute the quantum corrections to infinitely many polynomial relations.

We circumvent this difficulty by defining the algebra of the quantum invariants at one stroke as the subalgebra of $\mathcal{U}(\widehat{\Re})$ which is annihilated by a suitable derivation $\delta$ :

$$
\widehat{\mathfrak{h}}:=\operatorname{Ker} \delta \subset \mathcal{U}(\widehat{\mathfrak{R}})
$$

where $\delta$ maps $\mathcal{U}(\widehat{\mathfrak{R}})$ into another auxiliary algebra $\mathcal{U}(\mathfrak{C})$. This quantum condition is modelled after the classical condition (2.4), (2.5). The property of $\delta$ as a derivation ensures that its kernel $\operatorname{Ker} \delta$ is an associative, filtered algebra (Prop. 2). Then, it suffices to identify the quantum counterparts of the classical generating invariants as elements of Ker $\delta$, and verify that they generate Ker $\delta$ (the principle of correspondence, Prop. 4). From this knowledge one concludes that the correction terms in the quantum polynomial relations belong to Ker $\delta$, and thus are again quantum invariants, without the need to actually compute them. The principle of correspondence will be established (Prop. 4) in the form of linear isomorphisms between the classical spaces $\mathfrak{h}^{(l)}$ and the quotients of the filtered spaces $\widehat{\mathfrak{h}}^{(l)} / \widehat{\mathfrak{h}}^{(l-1)}$. 
These steps result in a consistent algebraic quantization of the reparametrization invariant observables of the closed bosonic string in any spacetime dimension. We shall show that they can be achieved provided the quadratic generation property is true.

Explicit calculations [1] in 1+3-dimensional space-time show complete agreement with the intrinsic approach of ref. [12] (with the intrinsically so far undetermined parameters taking specific values). In particular, the additional structures outlined in Sect. 2.5, $\{\mathfrak{A}, \mathfrak{A}\}=0$ and $\{\mathfrak{a}, \mathfrak{U}\} \subset \mathfrak{U}$, also hold as commutator relations in the quantum algebra $\widehat{\mathfrak{h}}$ as far as they could be checked, cf. also Sect. 7 and note added in proof.

\section{Construction of the quantum algebra}

We want to construct the quantum algebra of observables according to the strategy just outlined. In order to focus our attention on the relevant algebraic structures, we proceed from an abstract description of the Lie algebra $\mathfrak{R}$ and the classical Poisson algebra $\mathfrak{h}$ based on the results of [7]. It does not refer to the interpretation of the algebra elements as functionals of the string's world surface, but completely takes into account their algebraic aspects. Along the way, we provide the necessary details omitted in Sect. 2.

Let $e_{0}, \ldots, e_{D-1}$ be a basis of $\mathbb{R}^{D}$. A shuffle sum is a sum over basis vectors of $\otimes^{N} \mathbb{R}^{D}$ of the form

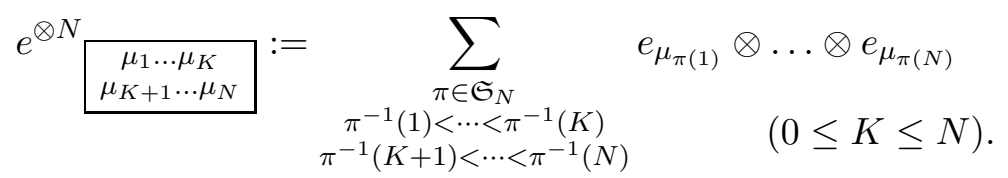

Let $V_{N}$ be the subspaces of $\otimes^{N} \mathbb{R}^{D}$ spanned by all shuffle sums with $1 \leq$ $K<N$, and $p_{N}: \otimes^{N} \mathbb{R}^{D} \rightarrow \otimes^{N} \mathbb{R}^{D} / V_{N}$ the canonical projections onto the quotient spaces $\otimes^{N} \mathbb{R}^{D} / V_{N}$. We identify the monodromy variables with the components of the tensors $\mathcal{R}_{\mu_{1} \ldots \mu_{N}}^{t}=p_{N}\left(e_{\mu_{1}} \otimes \ldots \otimes e_{\mu_{N}}\right)$, i.e., they satisfy the defining linear relations

$$
\mathcal{R}_{\substack{\mu_{1} \ldots \mu_{K} \\ \mu_{K+1} \ldots \mu_{N}}}^{t}=0 \quad(1 \leq K<N) .
$$

Their linear span $\mathfrak{R}=\bigoplus_{N=1}^{\infty} \otimes^{N} \mathbb{R}^{D} / V_{N}$ equipped with the bracket

$$
\begin{aligned}
& \left\{\mathcal{R}_{\mu}^{t}, \mathcal{R}_{\nu}^{t}\right\}=\left\{\mathcal{R}_{\mu_{1} \ldots \mu_{N}}^{t}, \mathcal{R}_{\nu}^{t}\right\}=0,
\end{aligned}
$$

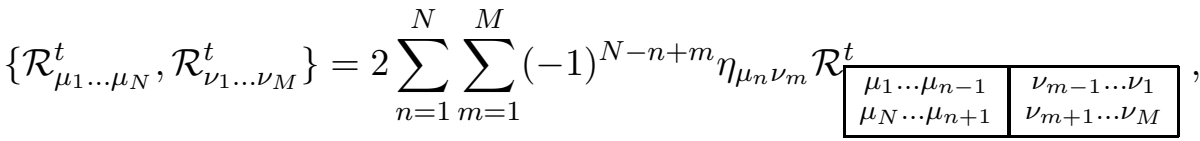


is an infinite-dimensional Lie algebra, graded with respect to the degree $l=N-2$. The second formula holds for $N \geq 2, M \geq 2$, and cannot be extended to $N=1$ or $M=1$ without violating the Jacobi identity. Instead, by the first formula, $\mathcal{R}_{\mu}^{t} \equiv \mathcal{P}_{\mu}$ are central elements of $\mathfrak{R}$.

The bracket canonically extends to the symmetric envelopping algebra $\mathcal{S}(\mathfrak{R})$ of $\mathfrak{R}$, which becomes a Poisson algebra. $\mathfrak{h} \subset \mathcal{S}(\mathfrak{R})$ is the subspace spanned by the polynomials (invariants) of the form

$$
\begin{aligned}
& \mathcal{Z}_{\mu_{1} \ldots \mu_{N}}=\sum_{K=1}^{N} \mathcal{Z}_{\mu_{1} \ldots \mu_{N}}^{(K)} \\
& \mathcal{Z}_{\mu_{1} \ldots \mu_{N}}^{(K)}=\frac{1}{K !} \mathfrak{z}_{N} \circ\left(\sum_{1 \leq a_{1}<\ldots<a_{K-1}<N} \mathcal{R}_{\mu_{1} \ldots \mu_{a_{1}}}^{t} \mathcal{R}_{\mu_{a_{1}+1} \ldots \mu_{a_{2}}}^{t} \cdots \mathcal{R}_{\mu_{a_{K-1}+1} \ldots \mu_{N}}^{t}\right)
\end{aligned}
$$

where $\mathfrak{z}_{N}$ denotes the sum over the cyclic permutations of the Lorentz indices. This space $\mathfrak{h}$ is in fact a Poisson subalgebra of $\mathcal{S}(\mathfrak{R})$.

We capture the characterization of the invariants by their reparametrization invariance, eqs. (2.2) and (2.4), with the help of the linear map $\partial$ which maps $\mathfrak{R}$ into $\mathbb{R}^{D} \otimes \mathfrak{R}$ by

$$
\partial\left(\mathcal{P}_{\mu}\right):=0, \quad \partial\left(\mathcal{R}_{\mu_{1} \ldots \mu_{N}}^{t}\right):=e_{\mu_{1}} \otimes \mathcal{R}_{\mu_{2} \ldots \mu_{N}}^{t}-e_{\mu_{N}} \otimes \mathcal{R}_{\mu_{1} \ldots \mu_{N-1}}^{t} .
$$

$\partial$ extends canonically to a derivation $\partial$ from the commutative algebra $\mathcal{S}(\mathfrak{R})$ into the commutative algebra $\mathcal{S}\left(\mathbb{R}^{D} \oplus \mathfrak{R}\right.$ ) (along the injection homomorphism from $\mathcal{S}(\mathfrak{R})$ into $\left.\mathcal{S}\left(\mathbb{R}^{D} \oplus \mathfrak{R}\right)\right)$. The invariants are exactly those elements of $\mathfrak{R}$ which are annihilated by $\partial$ :

$$
\mathfrak{h}=\operatorname{Ker}(\partial) \subset \mathcal{S}(\mathfrak{R})
$$

cf. (2.5).

This abstract description of the classical algebra of string observables is the starting point for its quantization. As the monodromy variables $\mathcal{R}_{\mu_{1} \ldots \mu_{N}}^{t}$ form a Lie algebra, they can easily be quantized by defining the commutator as $i \hbar$ times the Lie bracket $\{\cdot, \cdot\}$. We call this Lie algebra $\widehat{\Re}$. By the theorem of Poincaré-Birkhoff-Witt, the universal envelopping algebra $\mathcal{U}(\widehat{\Re})$ is an associative filtered algebra.

We want to parallel the prescription (4.6) by "quantizing" the derivation $\partial$. But whereas a linear map defined on a Lie algebra (considered as a linear space) extends canonically to a derivation on its symmetric envelopping algebra, the same is not true for an extension to the universal envelopping algebra, unless the map has the property of a derivation with respect to the Lie bracket. For this reason, we first need an extension of the Lie bracket on $\widehat{\mathfrak{R}}$ to a Lie bracket on the auxiliary space $\mathfrak{C}:=\mathbb{R}^{D} \oplus \widehat{\mathfrak{R}}$ by defining suitable 
commutation relations between the elements of $\widehat{\Re}$ and $\mathbb{R}^{D}$, with respect to which the "quantized" map $\delta: \widehat{\Re} \rightarrow \mathcal{U}(\mathfrak{C})$ is a derivation. Then $\delta$ extends to $\mathcal{U}(\widehat{\mathfrak{R}})$, and we can define the quantum algebra of observables $\widehat{\mathfrak{h}}$ as its kernel within $\mathcal{U}(\widehat{\mathfrak{R}})$.

Proposition 1: 1 . The bracket $[\cdot, \cdot]: \mathfrak{C} \times \mathfrak{C} \rightarrow \mathfrak{C}$ on the auxiliary space $\mathfrak{C}:=\mathbb{R}^{D} \oplus \widehat{\mathfrak{R}}$

$$
\begin{array}{ll}
{\left[\mathcal{R}_{I}^{t}, \mathcal{R}_{J}^{t}\right]:=i \hbar\left\{\mathcal{R}_{I}^{t}, \mathcal{R}_{J}^{t}\right\},} & {\left[e_{\mu}, e_{\nu}\right]:=0} \\
{\left[\mathcal{R}_{\mu_{1} \ldots \mu_{N}}^{t}, e_{\mu}\right]:=0 \quad(N \neq 2),} & {\left[\mathcal{R}_{\mu \nu}^{t}, e_{\kappa}\right]:=-2 i \hbar\left(\eta_{\nu \kappa} e_{\mu}-\eta_{\mu \kappa} e_{\nu}\right)}
\end{array}
$$

is a Lie bracket. As a Lie algebra, $\mathfrak{C}$ is a semidirect sum of $\widehat{\mathfrak{R}}$ with the abelian Lie algebra $\mathbb{R}^{D}$.

2. The linear map $\delta$ from $\widehat{\Re}$ into the universal envelopping algebra $\mathcal{U}(\mathfrak{C})$,

$$
\begin{aligned}
\delta\left(\mathcal{R}_{\mu}^{t}\right) & :=0 \\
\delta\left(\mathcal{R}_{\mu_{1} \ldots \mu_{N}}^{t}\right) & :=\frac{1}{2}\left[e_{\mu_{1}}, \mathcal{R}_{\mu_{2} \ldots \mu_{N}}^{t}\right]_{+}-\frac{1}{2}\left[e_{\mu_{N}}, \mathcal{R}_{\mu_{1} \ldots \mu_{N-1}}^{t}\right]_{+}(N \geq 2),
\end{aligned}
$$

where $[\cdot, \cdot]_{+}$denotes the anti-commutator in $\mathcal{U}(\mathfrak{C})$, is well-defined and has the property of a derivation:

$$
\delta([A, B])=[\delta(A), B]+[A, \delta(B)] \quad \forall A, B \in \mathfrak{R} .
$$

It extends canonically to a derivation $\delta: \mathcal{U}(\widehat{\mathfrak{R}}) \rightarrow \mathcal{U}(\mathfrak{C})$ (along the injection homomorphism).

Comment: Whereas the map $\delta$ is obviously a quantum analogue of a corresponding classical structure (the derivative $\partial_{\sigma}$, eq. (2.2), or equivalently the map $\partial$, eq. (4.5)), the same is not true for the extension of the Lie bracket. The canonical bracket between $\mathcal{R}_{\mu_{1} \ldots \mu_{N}}^{t}(\tau, \sigma)$ and $u_{\mu}(\tau, \sigma)$ is ill-defined and violates the Jacobi identity. Therefore, the success of our quantum prescription is a rather non-trivial feature.

Proof: 1 . As the monodromy variables with the bracket $\{\cdot, \cdot\}$ form a Lie algebra, it is sufficient to prove the Jacobi identity for multiple commutators involving the basis vectors of $\mathbb{R}^{D}$. This can be explicitly verified from the above definitions and the definition of the Poisson bracket, eq. (4.3).

2. $\delta$ is well-defined if it respects the linear dependencies (4.2) among the monodromy variables. This is verified by straightforward calculation using recursive relations for the shuffle sums following from their definition (4.1):

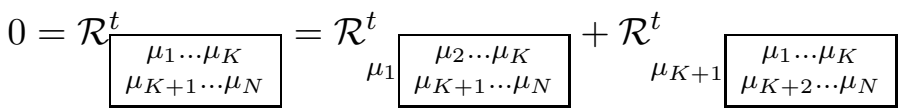

$$
\begin{aligned}
& =\mathcal{R}_{\begin{array}{c}
\mu_{1} \ldots \mu_{K-1} \\
\mu_{K+1} \ldots \mu_{N}
\end{array}}^{t} \mu_{K}+\mathcal{R}_{\begin{array}{c}
\mu_{1} \ldots \mu_{K} \\
\mu_{K+1} \ldots \mu_{N-1}
\end{array} \mu_{N}}^{t} .
\end{aligned}
$$


The derivation property of $\delta$ is obtained with a rather lengthy but equally straightforward calculation [1] from the definition (4.7) of the Lie bracket together with (4.10). The case $N=2$ has to be treated separately. Q.E.D.

As $\delta$ extends to a derivation on the universal envelopping algebra $\mathcal{U}(\widehat{\Re})$, products and commutators of any two elements in its kernel are also annihilated by $\delta$. Hence its kernel $\operatorname{Ker} \delta$ is a subalgebra of $\mathcal{U}(\widehat{\Re})$ :

Proposition 2: The kernel $\widehat{\mathfrak{h}}=\operatorname{Ker} \delta \subset \mathcal{U}(\widehat{\mathfrak{R}})$ of the derivation $\delta$ is an associative non-commutative subalgebra of $\mathcal{U}(\widehat{\mathfrak{R}})$.

It will be shown in the next section that $\widehat{\mathfrak{h}}$ is a filtered algebra and its filtration is compatible with the grading of the classical algebra $\mathfrak{h}$. Furthermore, it satisfies the principle of correspondence in the sense of an association between classical invariants and quantum invariants up to order $\hbar$, which respects both products and brackets.

\section{The Principle of Correspondence}

As discussed in section 2, the classical Poisson algebras of monodromy variables, $\mathcal{S}(\mathfrak{R})=\bigoplus_{l} \mathcal{S}(\mathfrak{R})^{(l)}$, and of invariants, $\mathfrak{h}=\bigoplus_{l} \mathfrak{h}^{(l)}$, are graded with respect to the degree $l \geq-1$, and

$$
\mathfrak{h}^{(l)}=\operatorname{Ker} \partial \cap \mathcal{S}(\mathfrak{R})^{(l)} .
$$

Since the underlying Lie algebra for the commutative envelopping algebra $\mathcal{S}(\mathfrak{R})$ is the same as for the non-commutative envelopping algebra $\mathcal{U}(\widehat{\Re})$ (except for the factor $i \hbar$ ), the latter exhibits a corresponding filtration $\mathcal{U}(\widehat{\Re})=\bigcup_{l} \mathcal{U}(\widehat{\Re})^{(l)}$ with $\mathcal{U}(\widehat{\Re})^{(l-1)} \subset \mathcal{U}(\widehat{\Re})^{(l)}$, cf. eq. (3.1). The subspaces $\mathcal{U}(\widehat{\Re})^{(l)}$ are spanned by all non-commutative monomials of order $K$ in components of $\mathcal{R}_{\mu_{1} \ldots \mu_{N}}^{t}$ with total tensor rank $N$ and $N-K-1 \leq l$.

Due to the fact that a commutator $[A, B]$ is of lower degree than the individual products $A B, B A$, the quotient spaces are naturally isomorphic to the classical spaces of degree $l$ in $\mathcal{S}(\mathfrak{R})$ :

$$
\mathcal{U}(\widehat{\Re})^{(l)} / \mathcal{U}(\widehat{\Re})^{(l-1)} \cong \mathcal{S}(\Re)^{(l)} .
$$

The corresponding statements hold also for the commutative and non-commutative envelopping algebras $\mathcal{S}\left(\mathbb{R}^{D} \oplus \mathfrak{R}\right)$ and $\mathcal{U}(\mathfrak{C})$, where we assign the degree $l=-1$ to the generators of $\mathbb{R}^{D}$. The derivation $\delta$ takes $\mathcal{U}(\widehat{\Re})^{(l)}$ to $\mathcal{U}(\mathfrak{C})^{(l-1)}$.

We denote by $\Pi^{(l)}$ the canonical projection from $\mathcal{U}(\widehat{\mathfrak{R}})^{(l)}$ to $\mathcal{S}(\mathfrak{R})^{(l)}$, and use the same symbol for its continuation to $\mathcal{U}(\mathfrak{C})^{(l)}$. In particular, applied 
to a non-commutative polynomial, $\Pi^{(l)}$ is insensitive to the order of factors. We have

Proposition 3: The following intertwining rules hold for all $A \in \mathcal{U}(\widehat{\Re})^{(l)}$, $B \in \mathcal{U}(\widehat{\mathfrak{R}})^{\left(l^{\prime}\right)}$

$$
\begin{aligned}
\Pi^{\left(l+l^{\prime}+1\right)}(A \cdot B) & =\Pi^{(l)}(A) \cdot \Pi^{\left(l^{\prime}\right)}(B), \\
\Pi^{\left(l+l^{\prime}\right)}([A, B]) & =i \hbar\left\{\Pi^{(l)}(A), \Pi^{\left(l^{\prime}\right)}(B)\right\}, \\
\Pi^{(l-1)} \circ \delta(A) & =\partial \circ \Pi^{(l)}(A) .
\end{aligned}
$$

Proof: Due to the parallelism between the very definitions of the classical and quantum operations (product, bracket, and derivation) on $\mathfrak{R}$ resp. $\widehat{\mathfrak{R}}$, their continuations to $\mathcal{S}(\mathfrak{R})$ resp. $\mathcal{U}(\widehat{\mathfrak{R}})$ differ only by the necessity of factor ordering for the latter. Thus, ignoring factor ordering and terms of lower degree (in particular commutators) by means of the projections, takes the quantum operations to the classical operations.

Q.E.D.

Because of its definition as the kernel of a derivation, the quantum algebra $\widehat{\mathfrak{h}}$ of invariants inherits the filtration, as anticipated in eq. (3.2):

$$
\widehat{\mathfrak{h}}=\bigcup_{l \geq-1} \widehat{\mathfrak{h}}^{(l)}, \quad \widehat{\mathfrak{h}}^{(l)}=\operatorname{Ker} \delta \cap \mathcal{U}\left(\widehat{\mathfrak{R}}^{(l)}\right.
$$

Corollary: The projections $\Pi^{(l)}$ map the spaces of quantum observables $\widehat{\mathfrak{h}}^{(l)}$ injectively into the spaces of classical observables $\mathfrak{h}^{(l)}$. Non-commutative multiplication and commutators are mapped to commutative multiplication and Poisson brackets.

Proof: By eq. (5.5), the image of $\widehat{\mathfrak{h}}^{(l)}=\operatorname{Ker} \delta \cap \mathcal{U}(\widehat{\mathfrak{R}})^{(l)}$ belongs to Ker $\partial \cap \mathcal{S}(\mathfrak{R})^{(l)}$ which equals $\mathfrak{h}^{(l)}$. The projection is injective on $\widehat{\mathfrak{h}}^{(l)}$ because it is injective on $\mathcal{U}(\widehat{\Re})^{(l)}$. The other statements are just reformulations of eqs. (5.3), (5.4).

Q.E.D.

Thus, if we show that $\Pi^{(l)}$ is also surjective onto $\mathfrak{h}^{(l)}$, we have linear isomorphisms between the classical and quantum observables, preserving the pertinent algebraic structures up to quantum corrections. Hence every element of $\widehat{\mathfrak{h}}$ corresponds to a classical invariant in leading order of $\hbar$.

Proposition 4: 1 . For each classical invariant $\mathcal{Z}_{\mu_{1} \ldots \mu_{N}}^{(2)}$ of polynomial order $K=2$, the corresponding quantum invariant defined by

$$
\widehat{\mathcal{Z}}_{\mu_{1} \ldots \mu_{N}}^{(2)}=\frac{1}{2} \mathfrak{z} N \circ\left(\sum_{1 \leq a<N} \mathcal{R}_{\mu_{1} \ldots \mu_{a}}^{t} \cdot \mathcal{R}_{\mu_{a+1} \ldots \mu_{N}}^{t}\right) \in \mathcal{U}(\widehat{\Re})^{(N-3)}
$$

lies in the kernel of derivation $\delta$. 
2. Thus, by the quadratic generation hypothesis, all classical generators have their quantum counterparts in $\widehat{\mathfrak{h}}$ given by eq. (5.7).

3. Using the generation of the classical algebra of observables, this defines linear assignments $\alpha^{(l)}: \mathfrak{h}^{(l)} \rightarrow \widehat{\mathfrak{h}}^{(l)}$, such that

$$
\Pi^{(l)} \circ \alpha=\mathrm{id},
$$

and all elements of the form

$$
\begin{array}{r}
\alpha^{\left(l+l^{\prime}+1\right)}(A \cdot B)-\alpha^{(l)}(A) \cdot \alpha^{\left(l^{\prime}\right)}(B) \\
\text { or } \quad \alpha^{\left(l+l^{\prime}\right)}(i \hbar\{A, B\})-\left[\alpha^{(l)}(A), \alpha^{\left(l^{\prime}\right)}(B)\right]
\end{array}
$$

with $A \in \mathfrak{h}^{(l)}, B \in \mathfrak{h}^{\left(l^{\prime}\right)}$, are annihilated by $\Pi^{\left(l+l^{\prime}+1\right)}$ and $\Pi^{\left(l+l^{\prime}\right)}$, respectively.

4. The projections $\Pi^{(l)}$ are surjective.

Proof: Statement 1 is shown to be true by straightforward calculation using the definition of the derivation $\delta$ and of the bracket $[\cdot, \cdot]$ between $\mathbb{R}^{D}$ and $\widehat{\mathfrak{R}}$. Statement 2 is obvious.

The assignment $\alpha$ is obtained as follows. Represent an element of $\mathfrak{h}^{(l)}$ as a polynomial in the generators and their multiple Poisson brackets, i.e., as an element of the Poisson algebra $\mathcal{S}(\mathfrak{F})$ (cf. Sect. 2.4), applying any convention regarding the addition of polynomial relations (of the same degree) from the ideal $\mathfrak{I}$. Replace in this polynomial every monomial by a corresponding product of multiple commutators of the quantum generators according to the previous statements, applying any convention regarding factor ordering. The resulting polynomial is an element of $\mathcal{U}(\widehat{\Re})^{(l)}$, and because $\delta$ is a derivation which annihilates the quantum generators, this polynomial is also annihilated by $\delta$ and belongs to $\widehat{\mathfrak{h}}^{(l)}$. Applying the projection $\Pi^{(l)}$ restores the classical original polynomial thanks to Prop. 3, proving eq. (5.8). In particular, all ambiguities due to the conventions above are annihilated by the projection, because commutators in $\widehat{\mathfrak{h}}^{(l)}$ belong to $\widehat{\mathfrak{h}}^{(l-1)}$, and because the polynomial relations hold in $\mathfrak{h}$. The other asserted properties of $\alpha^{(l)}$ are then fulfilled by construction, because the two terms to be compared just correspond to two different conventions for the assignment $\mathfrak{h}^{(l)} \rightarrow \widehat{\mathfrak{h}}^{(l)}$.

Eq. (5.8) implies surjectivity of $\Pi^{(l)}$ by $\mathfrak{h}^{(l)}=\Pi^{(l)}\left(\alpha^{(l)}\left(\mathfrak{h}^{(l)}\right)\right)$. Q.E.D.

With Prop. 4, we have achieved the principle of correspondence for the relation between the filtered quantum algebra $\widehat{\mathfrak{h}}$ and the graded classical algebra $\mathfrak{h}$.

Comment: The quantum invariants of order 2 are obtained as symmetrized quadratic polynomials in the quantum monodromy variables. The same holds not true for quantum invariants of higher order, since noncommutative readings of eq. (4.4) for $K>2$ in general do not belong to 
Ker $\delta$. Instead, quantum invariants of higher order must be defined through the generating property by the embeddings $\alpha^{(l)}$.

\section{The quadratic generation property}

The assumed validity of the quadratic generation property of the classical algebra $\mathfrak{h}$ (cf. Sect. 2.4) enters our construction of the quantum algebra only in Prop. 4. We want to emphasize that even if this hypothesis should fail, it would be sufficient for the construction if for any other system of generating invariants the quantum counterparts in Ker $\delta$ could be given.

By an argument given in [7, Sect. IV], the quadratic generation property would follow from a related property of the Lie algebra $\mathfrak{R}$ (given below). There is considerable evidence for the latter property to hold in any dimension, although partial general arguments given so far are not yet conclusive 11. 14. Explicit verification at least up to degree 7 is available in $1+3$ dimensions [12, p. 28].

The relevant property of $\mathfrak{R}$ is most conveniently formulated in the center of mass frame $\mathcal{P}_{\mu}=(m, 0, \ldots, 0)$ where there is an association between an algebraic basis of $\mathfrak{h}$ ("standard invariants") and the components of $\mathcal{R}_{\mu_{1} \ldots \mu_{N}}^{t}$ with $\mu_{1} \neq 0, \mu_{N} \neq 0$. The latter elements of $\mathfrak{R}$ span a Lie subalgebra $\mathfrak{R}_{0}$. The "exceptional" elements $\sum_{i} \mathcal{R}_{i 0 \ldots 0 i}^{t}$ of odd $\operatorname{rank} N=l+2$ cannot be generated by Poisson brackets within $\Re_{0}$ [7, Sect. IV]. ${ }^{3}$ The hypothesis is that the elements of $\mathfrak{R}_{0}$ of degree $l \leq 1$ together with the infinite series of exceptional elements generate $\mathfrak{R}_{0}$. Because the corresponding standard invariants are quadratic [7. Sect. IV], this property of $\mathfrak{R}_{0}$ entails the desired quadratic generation property for $\mathfrak{h}$.

Substantiating the expectation (spurred by extensive experience with this algebra) that this property of the classical algebra holds in general, would complete the construction of the quantum algebra in any dimension.

\section{$7 \quad$ Additional structures}

We include some partial results concerning the persistence in the quantum algebra $\mathfrak{h}$ of the classical structures $\{\mathfrak{A}, \mathfrak{A}\}=0$ and $\{\mathfrak{a}, \mathfrak{U}\} \subset \mathfrak{U}$, mentioned in Sect. 2.5. Although the survival of these additional structures in the quantum theory is highly desirable, it is not a prerequisite for the quantization of the string according to the prescription $\widehat{\mathfrak{h}}=\operatorname{Ker} \delta$.

\footnotetext{
${ }^{3}$ This circumstance explains the necessity of including, among the generators of $\mathfrak{h}$, the scalars $\mathcal{B}_{(l)}$ of odd degree $l$, which have a non-vanishing "leading" contribution proportional to the exceptional elements.
} 
We shall prove the quantum commutativity of the quadratic elements of the abelian subalgebra $\widehat{\mathfrak{A}}$ at any degree. These invariants are obtained from the generating functional $\operatorname{Tr}\left(\log \Phi_{\lambda \Gamma}\right)^{2}$ by variation with respect to the parameter $\lambda$, where the matrices $\Gamma^{\mu}$ satisfy the Clifford algebra $\Gamma^{\mu} \Gamma^{\nu}+$ $\Gamma^{\nu} \Gamma^{\mu}=2 \eta^{\mu \nu}$. We exploit the closed formula (2.3) for the Lie bracket on $\mathfrak{R}$ which we expect to be a convenient tool for further structural study.

The Clifford algebra implies the identity

$$
\left[\left[\Gamma_{\alpha}, \Gamma_{\beta}\right] \otimes\left[\Gamma^{\alpha}, \Gamma^{\beta}\right], \Gamma^{\mu} \otimes \mathbf{1}\right]=8\left[\Gamma_{\alpha} \otimes \Gamma^{\alpha}, \mathbf{1} \otimes \Gamma^{\mu}\right] .
$$

For $S^{\mu}=\lambda\left(\Gamma^{\mu} \otimes \mathbf{1}\right)$ and $T^{\mu}=\kappa\left(\mathbf{1} \otimes \Gamma^{\mu}\right)$, this identity entails

$$
\left[S \cdot T, S^{\mu}-T^{\mu}\right]=\left[W, S^{\mu}+T^{\mu}\right]
$$

with $W=\lambda \kappa \frac{\lambda^{2}+\kappa^{2}}{\lambda^{2}-\kappa^{2}}\left(\Gamma_{\alpha} \otimes \Gamma^{\alpha}\right)-\frac{1}{4} \frac{\lambda^{2} \kappa^{2}}{\lambda^{2}-\kappa^{2}}\left(\left[\Gamma_{\alpha}, \Gamma_{\beta}\right] \otimes\left[\Gamma^{\alpha}, \Gamma^{\beta}\right]\right)$. By the latter identity, the (otherwise unwieldy) differential term in the expression (2.3) for the bracket between the generating functionals becomes

$$
\begin{aligned}
\left.\operatorname{Tr}\left([W, X] \cdot \partial_{X}\right) \log \Phi_{X}\right|_{X=S+T} & =\left[W,\left.\log \Phi_{X}\right|_{X=S+T}\right] \\
& =\left[W, \log \Phi_{S}+\log \Phi_{T}\right],
\end{aligned}
$$

and (2.3) acquires the form without a differential term

$$
\begin{aligned}
& \left\{\log \Phi_{\lambda \Gamma}{ }^{\otimes}, \log \Phi_{\kappa \Gamma}\right\}= \\
& \quad=\left[W, \log \Phi_{\lambda \Gamma} \otimes \mathbf{1}+\mathbf{1} \otimes \log \Phi_{\kappa \Gamma}\right]-\left[V, \log \Phi_{\lambda \Gamma} \otimes \mathbf{1}-\mathbf{1} \otimes \log \Phi_{\kappa \Gamma}\right]
\end{aligned}
$$

with $V=\lambda \kappa\left(\Gamma_{\alpha} \otimes \Gamma^{\alpha}\right)$, and $W$ as above.

The expansion of the generating functional $\log \Phi_{\lambda \Gamma}$ simplifies considerably, because by virtue of the shuffle symmetries of the tensors $\mathcal{R}_{\mu_{1} \ldots \mu_{N}}^{t}$, only multiple commutators of Clifford matrices contribute to $\log \Phi_{\lambda \Gamma}$, which can be worked out [9, Sect. II]:

$$
\log \Phi_{\lambda \Gamma}=\mathbb{P}_{\mu}(\lambda) \Gamma^{\mu}+\mathbb{R}_{\mu \nu}(\lambda) \frac{1}{2}\left[\Gamma^{\mu}, \Gamma^{\nu}\right]
$$

where $\mathbb{P}_{\mu}$ and $\mathbb{R}_{\mu \nu}$ are certain $\mathfrak{R}$-valued power series in the parameter $\lambda$, involving only odd and even powers of $\lambda$, respectively. Hence

$$
\operatorname{Tr}\left(\log \Phi_{\lambda \Gamma}\right)^{2}=\mathbb{P}_{\mu}(\lambda) \mathbb{P}^{\mu}(\lambda)-2 \mathbb{R}_{\mu \nu}(\lambda) \mathbb{R}^{\mu \nu}(\lambda) .
$$

The Poisson bracket (7.4) between $\log \Phi_{\lambda \Gamma}$ and $\log \Phi_{\kappa \Gamma}$ can now be used to derive the brackets among the power series $\mathbb{P}_{\mu}$ and $\mathbb{R}_{\mu \nu}$. They read (after 
quantization)

$$
\begin{gathered}
{\left[\mathbb{P}_{\mu}(\lambda), \mathbb{P}_{\nu}(\kappa)\right]=-8 i \hbar \lambda^{3} \kappa^{3} \frac{\lambda^{-2} \mathbb{R}_{\mu \nu}(\lambda)-\kappa^{-2} \mathbb{R}_{\mu \nu}(\kappa)}{\lambda^{2}-\kappa^{2}}} \\
{\left[\mathbb{P}_{\tau}(\lambda), \mathbb{R}_{\mu \nu}(\kappa)\right]=-2 i \hbar \eta_{\tau \mu} \lambda^{3} \kappa^{2} \frac{\lambda^{-1} \mathbb{P}_{\nu}(\lambda)-\kappa^{-1} \mathbb{P}_{\nu}(\kappa)}{\lambda^{2}-\kappa^{2}}-(\mu \leftrightarrow \nu)} \\
{\left[\mathbb{R}_{\sigma \tau}(\lambda), \mathbb{R}_{\mu \nu}(\kappa)\right]=-2 i \hbar \eta_{\tau \mu} \lambda^{2} \kappa^{2} \frac{\mathbb{R}_{\sigma \nu}(\lambda)-\mathbb{R}_{\sigma \nu}(\kappa)}{\lambda^{2}-\kappa^{2}}-(\mu \leftrightarrow \nu)-(\sigma \leftrightarrow \tau) .}
\end{gathered}
$$

(The denominators always divide the power series in the numerator.)

Now, the quantum commutator between $\operatorname{Tr}\left(\log \Phi_{\lambda \Gamma}\right)^{2}$ and $\operatorname{Tr}\left(\log \Phi_{\kappa \Gamma}\right)^{2}$ given by eq. (7.6), can be evaluated with eq. (7.7). One finds that the resulting cubic terms again arrange into commutators, which can in turn be evaluated with eq. (17.7). The resulting quadratic terms once more arrange into commutators, which can be evaluated and finally vanish.

We conclude that the infinite series of quantized invariants generated by $\operatorname{Tr}\left(\log \Phi_{\lambda \Gamma}\right)^{2}$ commute among each other.

Unfortunately, this kind of argument fails for higher powers of $\log \Phi_{\lambda \Gamma}$, because the traces $\operatorname{Tr}\left(\log \Phi_{\lambda \Gamma}\right)^{K}$ do not belong to the kernel of $\delta$, and the general form of the necessary quantum corrections is not clear to us. The reason for the complication comes from the commutator in

$$
\delta \log \Phi_{\lambda \Gamma}=\lambda\left[e_{\mu} \Gamma^{\mu}, \log \Phi_{\lambda \Gamma}\right]
$$

which is both with respect to the Clifford matrices and with respect to the quantum operators in $\mathfrak{C}$, so that the matrix trace of a commutator does not vanish. Yet, we expect that there should be a likewise simple argument as in the classical case, giving rise to an equally large infinite system of commuting quantum observables.

The case of the semidirect action of $\mathfrak{a}$ on $\mathfrak{U}$ (cf. Sect. 2.5) appears much more difficult since it is still not well understood even at the classical level. It has been verified in the quantum algebra by explicit calculation in $1+3$ dimensions up to degree 4 [1] but a general proof is still lacking both for the quantum and the classical case (see note added in proof).

\section{Conclusion}

We have established a rather simple characterization of the quantum algebra of the gauge invariant observables of the closed bosonic string in any dimension, as the kernel of a suitable derivation

$$
\widehat{\mathfrak{h}}=\operatorname{Ker} \delta \subset \mathcal{U}(\widehat{\mathfrak{R}}) \text {. }
$$


$\delta$ is defined on the universal envelopping algebra $\mathcal{U}(\widehat{\mathfrak{R}})$ of an infinite-dimensional Lie algebra $\widehat{\Re}$ with values in $\mathcal{U}(\mathfrak{C})$ where $\mathfrak{C}$ is a Lie algebra extension of $\widehat{\mathfrak{R}}$. The only possible loophole in the argument is the lacking proof of an apparent structural property of the underlying classical theory, which is required for the proper correspondence with the quantum algebra.

In Sect. 7, we gave some first results which indicate that also the "additional structures" mentioned in Sect. 2.5 are respected by quantization.

The present algebraic definition of the quantum algebra bears some obvious resemblance with the BRST quantization scheme, where the derivation $\delta_{\mathrm{BRST}}$, given by the commutator with a BRST charge $Q$, determines the gauge invariant observables as its kernel. In the case at hand, however, $\delta$ is given just as a derivation which is not implemented as a commutator. This suffices to ensure that $\widehat{\mathfrak{h}}$ is an algebra. (It is also a ${ }^{*}$ algebra, if one treats the generators of the Lie algebras $\widehat{\mathfrak{R}}$ and $\mathfrak{C}$ as hermitean.)

We consider the quantized ambient algebras $\mathcal{U}(\widehat{\mathfrak{R}})$ and $\mathcal{U}(\mathfrak{C})$ only as auxiliary tools in order to establish the existence of $\widehat{\mathfrak{h}}$ with the desired prop-

erties. A representation of $\widehat{\mathfrak{h}}$ does not presuppose a representation of $\widehat{\mathfrak{R}}$, and in particular no "charge" operator which would implement $\delta$. We doubt the existence of such an object since $\delta$ as a map from $\mathcal{U}(\widehat{\Re})$ to $\mathcal{U}(\mathfrak{C})$ has no sensible extension to its image. It is likewise not meaningful to talk about $\delta$-invariant states.

The search for Hilbert space representations of the quantum string is therefore an independent problem. It should not be biased by any features of the ambient algebra $\mathcal{U}(\widehat{\mathfrak{R}})$ unless they are intrinsic to $\widehat{\mathfrak{h}}$. The construction (classification) of representations of $\widehat{\mathfrak{h}}$ is an exciting challenge for the future.

Acknowledgements. One of the authors (C.M.) wishes to thank Prof. K. Pohlmeyer, supervisor of her diploma thesis at the Physics Department of Freiburg University. Both of us are grateful for his critical comments on a preliminary version of the manuscript.

Note added in proof. A failure of the semidirect product structure $[\mathfrak{a}, \mathfrak{U}] \subset \mathfrak{U}$ in the intrinsic approach to quantization was reported recently in [18.

\section{References}

[1] C. Meusburger: "Die Quantisierung der Invariantenalgebra des Nambu-GotoStrings mit Hilfe einer konkreten Realisierung", diploma thesis (in German), Freiburg University, 2001

[2] M.B. Green, J.H. Schwarz, E. Witten: "Superstring Theory", Cambridge Univ. Press, 1987. 
[3] Y. Nambu: Lectures at the Copenhagen summer symposium 1970 (unpublished),

T. Goto: "Relativistic quantum mechanics of one-dimensional mechanical continuum and subsidiary condition of dual resonance model," Prog. Theor. Phys. 46 (1971) 1560.

[4] Y. Makeenko, A. A. Migdal: "Quantum Chromodynamics as dynamics of loops," Nucl. Phys. B 188 (1981) 269.

[5] A. M. Polyakov: "Gauge fields as rings of glue," Nucl. Phys. B 164 (1980) 171.

[6] K. Pohlmeyer: "A group theoretical approach to the quantization of the free relativistic closed string," Phys. Lett. B 119 (1982) 100.

[7] K. Pohlmeyer, K.-H. Rehren: "Algebraic properties of the invariant charges of the Nambu-Goto theory," Commun. Math. Phys. 105 (1986) 593.

[8] K. Pohlmeyer, K.-H. Rehren: "The algebra formed by the invariant charges of the Nambu-Goto theory: identification of a maximal abelian subalgebra," Commun. Math. Phys. 114 (1988) 55.

[9] K. Pohlmeyer, K.-H. Rehren: "The invariant charges of the Nambu-Goto theory: their geometric origin and their completeness," Commun. Math. Phys. 114 (1988) 177.

[10] K. Pohlmeyer: "The Poisson algebra of the invariant charges of the NambuGoto theory: Casimir elements," Commun. Math. Phys. 114 (1988) 351.

[11] K. Pohlmeyer: "Uncovering the detailed structure of the algebra formed by the invariant charges of closed bosonic strings moving in (1+2)-dimensional Minkowski space," Commun. Math. Phys. 163 (1994) 629.

[12] K. Pohlmeyer: "The Nambu-Goto theory of closed bosonic strings moving in 1+3-dimensional Minkowski space: The quantum algebra of observables," Annalen Phys. 8 (1999) 19.

[13] G. Handrich, C. Nowak: "The Nambu-Goto theory of closed bosonic strings moving in 1+3-dimensional Minkowski space: The construction of the quantum algebra of observables up to degree five," Annalen Phys. 8 (1999) 51.

[14] K. Pohlmeyer, "The Poisson algebra formed by the invariant charges of the Nambu-Goto theory of closed strings moving in $(1+2)$-dimensional Minkowski space: The derived algebra," Freiburg preprint THEP-91-1 (unpublished).

[15] D. Bahns: "Die Invariantenalgebra des Nambu-Goto-Strings in Erzeugungsund Vernichtungsoperatoren", diploma thesis (in German), Freiburg University, 1999.

[16] C. Kimstedt and K.-H. Rehren, 1983 (unpublished notes).

[17] G. Handrich: "Lorentz covariance for the quantum algebra of observables: Nambu-Goto strings in 3+1 dimensions", Int. J. Mod. Phys. A 17 (2002) 2331.

[18] G. Handrich, C. Paufler, J.B. Tausk, M. Walter: "The presentation of the quantum algebra of observables of the closed bosonic string in $1+3$ dimensions: the exact quantized generating relations of orders $\hbar^{6}$ and $\hbar^{7}$ ", Mod. Phys. Lett. A 17 (2002) 2611. 